\title{
Directional Stability and Controllability of Multi-Articulated Vehicles*
}

\author{
Akira AOKI**, Yoshitaka MARUMO** and Ichiro KAGEYAMA** \\ ${ }^{* *}$ College of Industrial Technology, Nihon University, \\ 1-2-1 Izumi-cho, Narashino-shi, Chiba 275-8575, Japan \\ E-mail: aaoki@maple.ocn.ne.jp
}

\begin{abstract}
This paper presents theoretical research in the fundamentals on the directional stability and controllability of multi-articulated vehicles. Analyses and numerical calculations are conducted by using models incorporating vehicle side-slipping and yawing. Two vehicle combinations are examined with regard to straight running stability, as well as steering sensitivity and off-tracking in steady-state turning. Type I is a tractor and two semitrailers; type II is a tractor, semitrailer, and full-trailer. The following conclusions are drawn. (1) The criterion of non-oscillatory stability of types I and II is a function of the stability factor as well as that of tractor and semitrailer combinations. (2) Type I is more stable than type II in oscillatory modes. (3) Type I differs slightly from type II regarding non-oscillatory stability, steering sensitivity, and off-tracking in steady-state turning.
\end{abstract}

Key words: Directional Stability, Controllability, Multiple Articulated Vehicles, Semitrailer, Full Trailer, Stability Factor, Steering Sensitivity, Oscillatory Mode, Off Tracking, Steady State Turning

\section{Introduction}

Two kinds of commercial articulated vehicles, tractor-semitrailer and truck-full-trailer combinations, have been used in Japan. Tractor-semitrailers are more common in Japan, whereas truck-full-trailers have become popular in Europe. In Australia, Canada and the USA, multi-articulated vehicles, called road trains, prevail.

Vehicle combinations are composed of a tractor unit and an arbitrary number of trailers; therefore, their lateral motion differs from that of unit vehicles. Most research on the lateral dynamics of commercial articulated vehicles has concentrated on tractor-semitrailer and truck-full-trailer combinations ${ }^{(1),(2)}$. Fewer studies have focused on multi-articulated vehicles used to transport large quantities of cargo.

The objective of this study is to examine multi-articulated vehicles, such as tractor-semitrailer-semitrailer (type I Double) and tractor-semitrailer-full-trailer (type II Double) combinations, in connection with lateral dynamics, on the assumption that they will be introduced into Japan. The fundamentals of directional stability and controllability of these vehicle combinations are addressed by analyses and numerical calculations using simplified mathematical models.

\section{Multi-articulated Vehicle Model}

\subsection{Hypothesis}

Simplified linear planar models are adopted with regard to the fixed right-hand 
rectangular coordinate system in a moving tractor (Fig. 1). In these models, angular displacement $\phi$ is small; therefore, $\sin \phi=\phi$ and $\cos \phi=1$, and products of each variable can be neglected because of very small values. Each multiple axle is reduced to a single axle, and the summation of cornering stiffness of all wheels of each multiple or single axle is treated as that of the wheel of the axle.

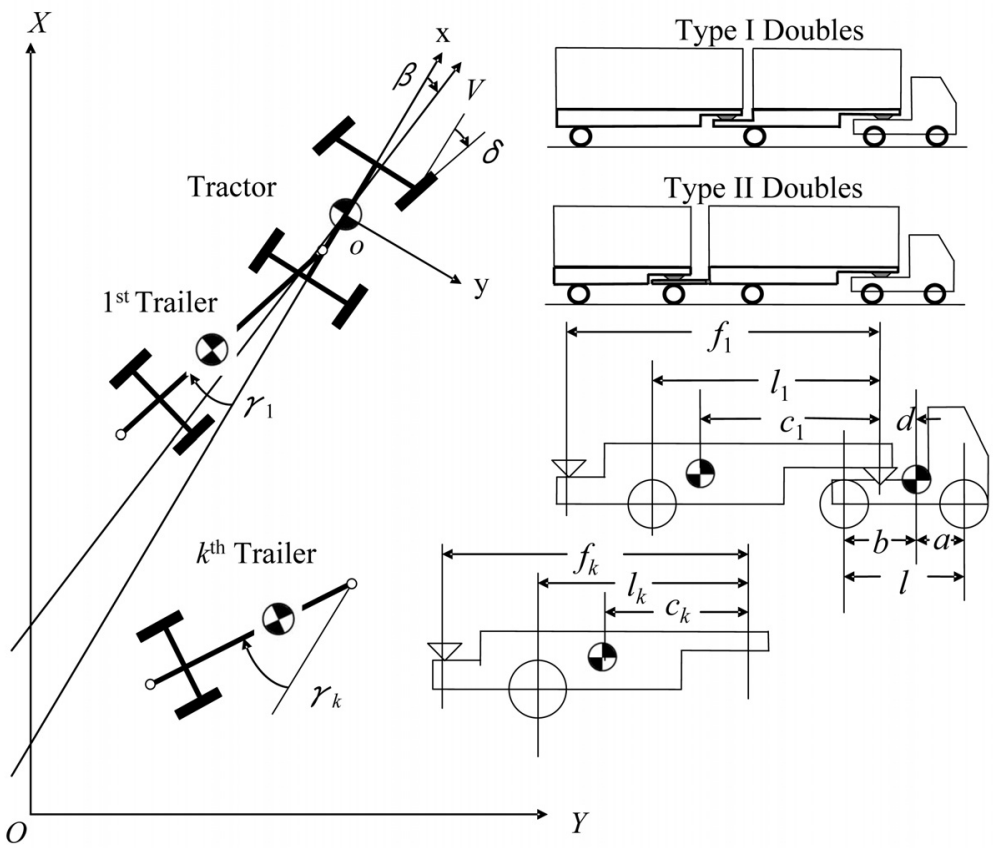

Fig. 1 Multi-articulated vehicle model

\subsection{Nomenclatures}

$a \quad$ : Distance between center of front axle and center of gravity of tractor

$b \quad$ : Distance between center of gravity and center of rear axle of tractor

$c_{k} \quad$ : Distance between front articulation point and center of gravity of $k^{\text {th }}$ trailer

$d \quad$ : Distance between center of gravity and articulation point of tractor

$f_{k} \quad$ : Distance between front and rear articulation points of $k^{\text {th }}$ trailer

$l \quad$ : Distance between center of front and rear axles of tractor

$l_{k} \quad:$ Distance between front articulation point and center of axle of $k^{\text {th }}$ trailer

$m$ : Mass of tractor

$m_{k}:$ Mass of $k^{\text {th }}$ trailer

$I \quad$ : Yawing moment of inertia of tractor

$I_{k} \quad$ : Yawing moment of inertia of $k^{\text {th }}$ trailer

$C_{f} \quad$ : Cornering stiffness of front wheel of tractor

$C_{r} \quad$ : Cornering stiffness of rear wheel of tractor

$C_{k} \quad$ : Cornering stiffness of $k^{\text {th }}$ trailer wheel

$P_{f} \quad$ : Vertical load of front axle of tractor

$P_{r} \quad$ : Vertical load of rear axle of tractor

$P_{c k} \quad$ : Vertical load of front articulation point of $k^{\text {th }}$ trailer

$P_{k} \quad:$ Vertical load of $k^{\text {th }}$ trailer axle

$\beta \quad$ : Side slip angle of center of gravity of tractor

$\beta_{f} \quad$ : Side slip angle of front wheel of tractor

$\beta_{r} \quad$ : Side slip angle of rear wheel of tractor

$\beta_{k} \quad$ : Side slip angle of $k^{\text {th }}$ trailer wheel

$\gamma_{k} \quad$ : Relative yawing angle between tractor and $k^{\text {th }}$ trailer 
$\delta \quad$ : Front steering angle of tractor

$r \quad$ : Yawing angular velocity of tractor

$V \quad$ : Forward velocity of vehicle combination

$g$ : Gravitational acceleration

$n \quad$ : Number of trailers

\subsection{Equations of Motion}

Equations (1), (2), and (3) are derived after the articulation point force is canceled and the cornering stiffness multiplied by the wheel sideslip angle is substituted into the wheel lateral force in the equilibrium equations of the $\mathrm{x}$ direction forces, the $\mathrm{y}$ direction forces, and yawing moments of each vehicle at constant running speed. In these equations, $n=0$ for tractors or trucks, $n=1$ for tractor-semitrailer combinations, $n=2$ for truck-full-trailer combinations or type I Doubles, and $n=3$ for type II Doubles.

$$
\begin{aligned}
& \left(m+\sum_{i=1}^{n} m_{i}\right) V \dot{\beta}+\left(C_{f}+C_{r}+\sum_{i=1}^{n} C_{i}\right) \beta \\
& -\sum_{j=1}^{n} m_{j}\left(d+\sum_{i=1}^{j-1} f_{i}+c_{j}\right) \dot{r}-\left[\left(b C_{r}-a C_{f}\right)+\sum_{j=1}^{n} C_{j}\left(d+\sum_{i=1}^{j-1} f_{i}+l_{j}\right)-\left(m+\sum_{i=1}^{n} m_{i}\right) V^{2}\right] r / V \\
& -\sum_{j=1}^{n}\left(m_{j} c_{j}+\sum_{i=j+1}^{n} m_{i} f_{j}\right) \ddot{\gamma}_{j}-\sum_{j=1}^{n}\left(l_{j} C_{j}+f_{j} \sum_{i=j+1}^{n} C_{i}\right) \dot{\gamma}_{j} / V-\sum_{j=1}^{n} C_{j} \gamma_{j}=C_{f} \delta . \\
& m d V \dot{\beta}+\left[(a+d) C_{f}+(d-b) C_{r}\right] \beta \\
& +I \dot{r}+\left[a(a+d) C_{f}-b(d-b) C_{r}+m d V^{2}\right] r / V=(a+d) C_{f} \delta . \\
& -\left(m_{k} c_{k}+\sum_{i=k+1}^{n} m_{i} f_{k}\right) V \dot{\beta}-\left(l_{k} C_{k}+f_{k} \sum_{i=k+1}^{n} C_{i}\right) \beta \\
& +\left[I_{k}+m_{k} c_{k}\left(d+\sum_{i=1}^{k-1} f_{i}+c_{k}\right)+\sum_{j=k+1}^{n} m_{j}\left(d+\sum_{i=1}^{j-1} f_{i}+c_{j}\right) f_{k}\right] \dot{r} \\
& +\left[l_{k} C_{k}\left(d+\sum_{i=1}^{k-1} f_{i}+l_{k}\right)+f_{k} \sum_{j=k+1}^{n} C_{j}\left(d+\sum_{i=1}^{j-1} f_{i}+l_{j}\right)\right] r / V-\left(m_{k} c_{k}+\sum_{i=k+1}^{n} m_{i} f_{k}\right) V r \\
& +\sum_{j=1}^{k-1}\left(m_{k} c_{k}+\sum_{i=k+1}^{n} m_{i} f_{k}\right) f_{j} \ddot{\gamma}_{j}+\left(I_{k}+m_{k} c_{k}^{2}+\sum_{i=k+1}^{n} m_{i} f_{k}^{2}\right) \ddot{\gamma}_{k}+\sum_{j=k+1}^{n}\left(m_{j} c_{j}+\sum_{i=j+1}^{n} m_{i} f_{j}\right) f_{k} \ddot{\gamma}_{j} \\
& +\sum_{j=1}^{k-1}\left(l_{k} C_{k}+f_{k} \sum_{i=k+1}^{n} C_{i}\right) f_{j} \dot{\gamma}_{j} / V+\left(l_{k}^{2} C_{k}+f_{k}^{2} \sum_{i=k+1}^{n} C_{i}\right) \dot{\gamma}_{k} / V+f_{k} \sum_{j=k+1}^{n}\left(l_{j} C_{j}+f_{j} \sum_{i=j+1}^{n} C_{i}\right) \dot{\gamma}_{j} / V \\
& +l_{k} C_{k} \gamma_{k}+f_{k} \sum_{i=k+1}^{n} C_{i} \gamma_{i}=0 .
\end{aligned}
$$

The sideslip angles of each wheel are given as follows.

$$
\begin{aligned}
& \beta_{f}=\delta-\beta-a r / V, \\
& \beta_{r}=-\beta+b r / V, \\
& \beta_{k}=-\beta+\left(d+\sum_{i=1}^{k-1} f_{i}+l_{k}\right) r / V+\sum_{i=1}^{k-1} f_{i} \dot{\gamma}_{i} / V+l_{k} \dot{\gamma}_{k} / V+\gamma_{k} .
\end{aligned}
$$

\section{Directional Stability}

\subsection{Characteristic Equations}

The directional stability of vehicle combinations in the straight running condition of 
fixed steering can be examined using characteristic equations obtained from equations of motion. Equations (1), (2), and (3) are transformed using Laplace operator $s$ as

$$
\left[\begin{array}{l}
\mathrm{B}(s) \\
R(s) \\
\Gamma_{1}(s) \\
\Gamma_{2}(s) \\
\bullet
\end{array}\right]=F(s)^{-1}\left[\begin{array}{l}
d_{100} \\
d_{200} \\
0 \\
0 \\
0
\end{array}\right] \Delta(s),
$$

where all initial values are zero, and

$$
F(s)=\left[\begin{array}{ccccc}
a_{110} s+a_{100} & b_{110} s+b_{100} & c_{121} s^{2}+c_{111} s+c_{101} & c_{122} s^{2}+c_{112} s+c_{102} & \bullet \\
a_{210} s+a_{200} & b_{210} s+b_{200} & 0 & 0 & 0 \\
a_{310} s+a_{300} & b_{310} s+b_{300} & c_{321} s^{2}+c_{311} s+c_{301} & c_{322} s^{2}+c_{312} s+c_{302} & \bullet \\
a_{410} s+a_{400} & b_{410} s+b_{400} & c_{421} s^{2}+c_{411} s & c_{422} s^{2}+c_{412} s+c_{402} & \bullet \\
\bullet & \bullet & \bullet & \bullet & \bullet
\end{array}\right] .
$$

Therefore, characteristic equations of multi-articulated vehicles are given as

$$
\operatorname{det}[F(s)]=\sum_{i=0}^{2 n+2} k_{i} s^{i}=0
$$

where $k_{i}$ is the coefficient of characteristic equations.

\subsection{Criterion of Directional Stability}

Typical stability criteria based on high-order algebraic equations are the Hurwitz criterion regarding the relationship between the root and coefficient of characteristic equations and the root locus method. This study analyzes non-oscillatory stability with regard to the coefficient of Eq. (7), and calculates roots of motion modes for changing forward velocity.

First, the sign of highest-order coefficient $k_{6}$ of type I Doubles $(n=2)$ is independent of forward velocity and is positive (Appendix 1). For $k_{0}<0$, Eq. (7) has a positive root at least because of $\operatorname{det}[F(0)]=k_{0}<0$ and $\operatorname{det}[F(+\infty)]=+\infty$. Therefore, $k_{0}=0$ is the limitation of non-oscillatory stability. The coefficient $k_{0}$ of type I Doubles is given as

$$
\begin{aligned}
& k_{0}=\left|\begin{array}{cccc}
a_{100} & b_{100} & c_{101} & c_{102} \\
a_{200} & b_{200} & 0 & 0 \\
a_{300} & b_{300} & c_{301} & c_{302} \\
a_{400} & b_{400} & 0 & c_{402}
\end{array}\right| \\
& =l^{2} l_{1} l_{2} C_{f} C_{r} C_{1} C_{2} / V+l l_{1} l_{2} C_{f} C_{r} C_{1} C_{2}\left(P_{f} / C_{f}-P_{r} / C_{r}\right) V / g \\
& =l^{2} l_{1} l_{2} C_{f} C_{r} C_{1} C_{2}\left(1+K V^{2}\right) / V .
\end{aligned}
$$

where $K$ is the stability factor as the index of the steering property, and

$$
\begin{aligned}
& K=\left(P_{f} / C_{f}-P_{r} / C_{r}\right) / g l, \\
& P_{f}=(b / l) m g+[(b-d) / l] P_{c 1}, \\
& P_{r}=(a / l) m g+[(a+d) / l] P_{c 1}, \\
& P_{c 1}=\left(1-c_{1} / l_{1}\right) m_{1} g+\left(1-f_{1} / l_{1}\right) P_{c 2}, \\
& P_{c 2}=\left(1-c_{2} / l_{2}\right) m_{2} g .
\end{aligned}
$$


The coefficient $k_{0}$ of type I Doubles is an expression similar to that of tractor and semitrailer combinations ${ }^{(3)}$.

Next, the sign of highest-order coefficient $k_{8}$ of type II Doubles $(n=3)$ is also independent of forward velocity and is positive, as with type I Doubles, and $k_{0}=0$ is the limitation of non-oscillatory stability. The coefficient $k_{0}$ of type II Doubles is given as

$$
k_{0}=l^{2} l_{1} l_{2} l_{3} C_{f} C_{r} C_{1} C_{2} C_{3}\left(1+K V^{2}\right) / V \text {. }
$$

Equation (11) is the vertical load of the articulation points modified and added to Eq. (9).

$$
\begin{aligned}
& P_{c 2}=\left(1-c_{2} / l_{2}\right) m_{2} g+\left(1-f_{2} / l_{2}\right) P_{c 3}, \\
& P_{c 3}=\left(1-c_{3} / l_{3}\right) m_{3} g .
\end{aligned}
$$

\section{Controllability in Steady-state Turning}

\subsection{Steady-state Turning Response}

The response of type I Doubles in steady-state turning is derived from Eqs. (1), (2), and (3) under the condition of Eq. (12) as Eq. (13), where $R$ is the steady-state turning radius; therefore, $r=V / R$.

$$
\begin{aligned}
& \dot{\beta}=\dot{r}=\ddot{\gamma}_{1}=\ddot{\gamma}_{2}=\dot{\gamma}_{1}=\dot{\gamma}_{2}=0 . \\
& {\left[\begin{array}{l}
\delta \\
\beta \\
\gamma_{1} \\
\gamma_{2}
\end{array}\right]=\left[\begin{array}{cccc}
-d_{100} & a_{100} & c_{101} & c_{102} \\
-d_{200} & a_{200} & 0 & 0 \\
0 & a_{300} & c_{301} & c_{302} \\
0 & a_{400} & 0 & c_{402}
\end{array}\right]^{-1}\left[\begin{array}{l}
-b_{100} \\
-b_{200} \\
-b_{300} \\
-b_{400}
\end{array}\right] V / R .}
\end{aligned}
$$

Equation (13) is reduced as

$$
\begin{aligned}
& \delta=\left(1+K V^{2}\right) l / R, \\
& \beta=b / R-\left(P_{r} / C_{r}\right) V^{2} / g R, \\
& \gamma_{1}=-\left(d-b+l_{1}\right) / R+\left(P_{1} / C_{1}-P_{r} / C_{r}\right) V^{2} / g R, \\
& \gamma_{2}=-\left(d-b+f_{1}+l_{2}\right) / R+\left(P_{2} / C_{2}-P_{r} / C_{r}\right) V^{2} / g R,
\end{aligned}
$$

where

$$
\begin{aligned}
& P_{1}=\left(c_{1} / l_{1}\right) m_{1} g+\left(f_{1} / l_{1}\right) P_{c 2}, \\
& P_{2}=\left(c_{2} / l_{2}\right) m_{2} g .
\end{aligned}
$$

The kinematic turning angles are given by substituting 0 into $V$ in Eq. (14) as

$$
\begin{aligned}
& \bar{\delta}=l / R, \\
& \bar{\beta}=b / R, \\
& \bar{\gamma}_{1}=-\left(d-b+l_{1}\right) / R, \\
& \bar{\gamma}_{2}=-\left(d-b+f_{1}+l_{2}\right) / R .
\end{aligned}
$$

The response of type II Doubles in steady-state turning is derived in much the same 
way as that of type I Doubles. Equation (17) is the group of equations modified and added to Eqs. (14), (15), and (16).

$$
\begin{aligned}
& \gamma_{3}=-\left(d-b+f_{1}+f_{2}+l_{3}\right) / R+\left(P_{3} / C_{3}-P_{r} / C_{r}\right) V^{2} / g R, \\
& P_{2}=\left(c_{2} / l_{2}\right) m_{2} g+\left(f_{2} / l_{2}\right) P_{c 3}, \\
& P_{3}=\left(c_{3} / l_{3}\right) m_{3} g, \\
& \bar{\gamma}_{3}=-\left(d-b+f_{1}+f_{2}+l_{3}\right) / R .
\end{aligned}
$$

\subsection{Steering Sensitivity}

The steering sensitivity of types I and II Doubles in steady-state turning is defined using each first equation of Eqs. (14) and (16) as

$$
\bar{\delta} / \delta=1 /\left(1+K V^{2}\right)
$$

Equation (18) is a function of the stability factor of Doubles and can be related to non-oscillatory stability as well as unit vehicles and tractor-semitrailer combinations. The steering property in $K=0$ is neutral steer, and the steering sensitivity is 1 , the same as kinematic turning. That in $K>0$ is understeer and the steering sensitivity is less than 1; and that in $K<0$ is oversteer, and the steering sensitivity is more than 1 . The coefficient $k_{0}$ in Eqs. (8) and (10) in $K \geq 0$ is independent of forward velocity and is positive; therefore, the non-oscillatory mode of vehicle combinations becomes stable. In contrast, the coefficient $k_{0}$ in $K<0$ is negative beyond the critical velocity $V_{c r}$ indicated in Eq. (19); therefore, it becomes unstable.

$$
V_{c r}=\sqrt{-1 / K}
$$

\subsection{Off-tracking}

The radii $R_{f}, R_{r}, R_{1}$ and $R_{2}$ of each center of the axle of type I Doubles in kinematic steady-state turning are given as

$$
\begin{aligned}
& R_{f}=\sqrt{R^{2}-b^{2}+l^{2}}, \\
& R_{r}=\sqrt{R^{2}-b^{2}}, \\
& R_{1}=\sqrt{R^{2}-b^{2}+(b-d)^{2}-l_{1}^{2}}, \\
& R_{2}=\sqrt{R^{2}-b^{2}+(b-d)^{2}+\left(f_{1}-l_{1}\right)^{2}-l_{1}^{2}-l_{2}^{2}} .
\end{aligned}
$$

Next, the radii in the steady-state turning having a forward velocity $V$ consist of the kinematic radii and the fluctuations depending on $V$. Those fluctuations can be expressed using the deviations from the kinematic turning radii as

$$
\begin{aligned}
& \Delta R_{f}=-a(\bar{\beta}-\beta)=-a\left(P_{r} / C_{r}\right) V^{2} / g R, \\
& \Delta R_{r}=b(\bar{\beta}-\beta)=b\left(P_{r} / C_{r}\right) V^{2} / g R, \\
& \Delta R_{1}=\left(d+l_{1}\right)(\bar{\beta}-\beta)-l_{1}\left(\bar{\gamma}_{1}-\gamma_{1}\right) \\
& =\left[d\left(P_{r} / C_{r}\right)+l_{1}\left(P_{1} / C_{1}\right)\right] V^{2} / g R, \\
& \Delta R_{2}=\left(d+f_{1}+l_{2}\right)(\bar{\beta}-\beta)-f_{1}\left(\bar{\gamma}_{1}-\gamma_{1}\right)-l_{2}\left(\bar{\gamma}_{2}-\gamma_{2}\right) \\
& =\left[d\left(P_{r} / C_{r}\right)+f_{1}\left(P_{1} / C_{1}\right)+l_{2}\left(P_{2} / C_{2}\right)\right] V^{2} / g R .
\end{aligned}
$$

The deviations of each center of an axle of type I Doubles from the turning radius of the center of gravity of the tractor are given as 


$$
\begin{aligned}
& \xi_{h}=R_{h}+\Delta R_{h}-R, \\
& h=f, r, 1,2 .
\end{aligned}
$$

Consequently, the maximum deviation among the tracks of each center of an axle is given as

$$
\begin{aligned}
& e_{s}=\left|\xi_{i}-\xi_{j}\right|_{\max }, \\
& i, j=f, r, 1,2(i \neq j) .
\end{aligned}
$$

The off-tracking of type II Doubles in steady-state turning is derived in the same manner as that of Type I Doubles. Equation (24) is the group of equations modified and added to Eqs. (20) and (21).

$$
\begin{aligned}
& R_{3}=\sqrt{R^{2}-b^{2}+(b-d)^{2}+\left(f_{1}-l_{1}\right)^{2}+\left(f_{2}-l_{2}\right)^{2}-l_{1}^{2}-l_{2}^{2}-l_{3}^{2}}, \\
& \Delta R_{3}=\left[d\left(P_{r} / C_{r}\right)+f_{1}\left(P_{1} / C_{1}\right)+f_{2}\left(P_{2} / C_{2}\right)+l_{3}\left(P_{3} / C_{3}\right)\right] V^{2} / g R .
\end{aligned}
$$

In addition, $\xi_{h}$ and $e_{s}$ of type II Doubles are obtained by adding $h=3$ and $i, j=3$ in Eqs. (22) and (23).

\section{Numerical Calculations}

\subsection{Vehicle Specifications}

The values of vehicle parameters used in numerical calculations are presented in Appendix 2. The wheel load divided by cornering stiffness of truck tires is nearly proportional to the wheel load ${ }^{(3)}$; therefore, the cornering stiffness can be obtained from the regression line with regard to this relationship.

\subsection{Characteristic Roots}

Figure 2 indicates the characteristic roots of type I Doubles in a standard condition as maximum loading. Figure 2, as well as subsequent figures, draws the roots of various forward velocities and particular damping ratio lines as the index of degree of stability in the second quadrant.

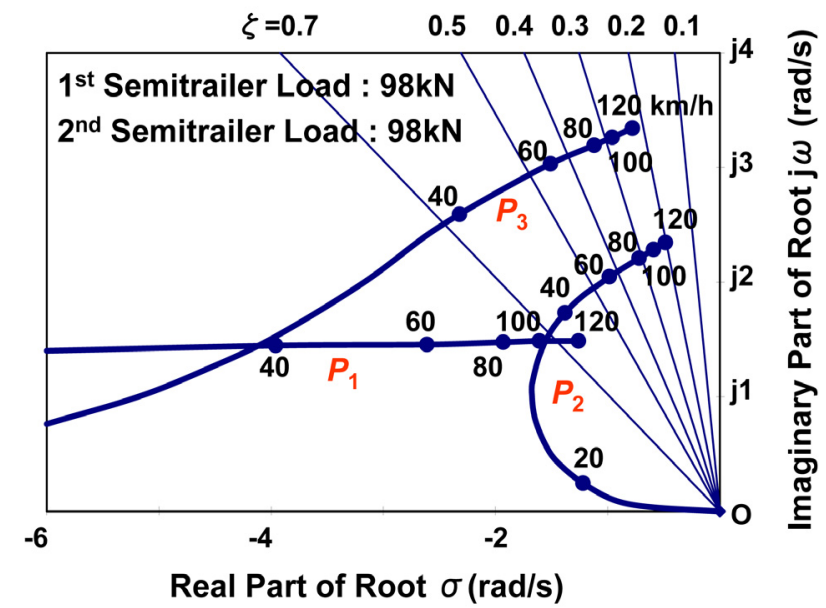

Fig. 2 Roots of type I Doubles (standard loading condition) 
The motion modes of type I Doubles consist of $P_{1}, P_{2}$, and $P_{3}$ in Fig. 2. The damped natural angular frequency of $P_{1}$ is almost constant in a wide range of forward velocities, and the damping ratio of $P_{1}$ is very large; therefore, the relative degree of stability is high. With forward velocity, the damped natural angular frequencies of $P_{2}$ and $P_{3}$ increase, while their damping ratio decreases. The damped natural angular frequency of $P_{3}$ exceeds that of $P_{2}$, and the damping ratio of $P_{2}$ and $P_{3}$ is similar in the forward velocity range of 40 to $120 \mathrm{~km} / \mathrm{h}$.

Figure 3 plots the characteristic roots of type II Doubles in a standard condition as maximum loading. Figure 3 and subsequent figures plot the real roots on lines parallel to the real axis.

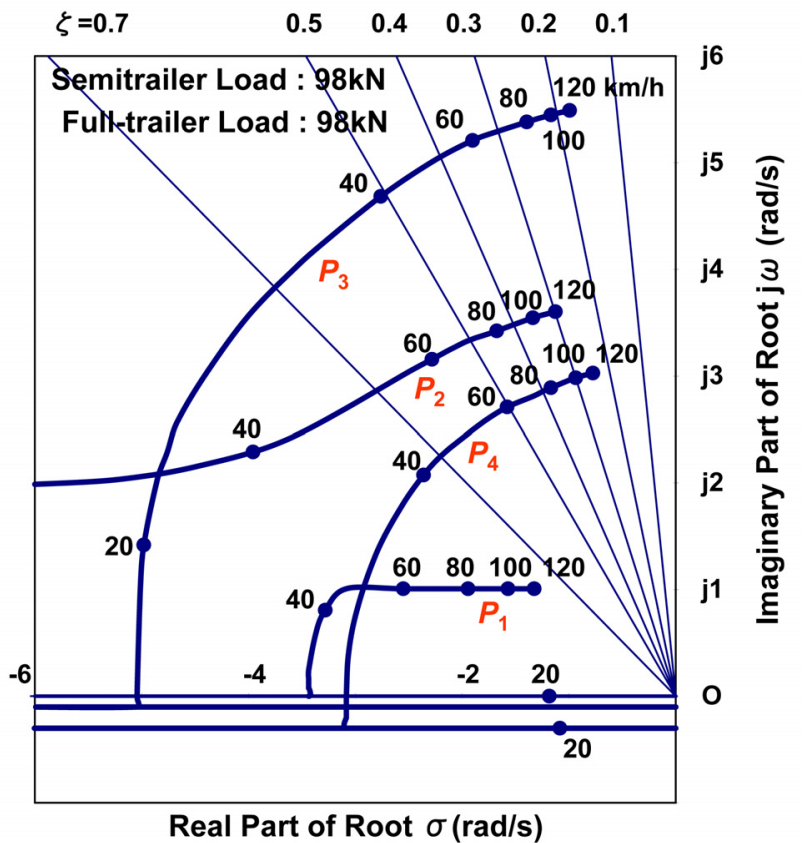

Fig. 3 Roots of type II Doubles (standard loading condition)

The motion modes of type II Doubles are $P_{1}, P_{2}, P_{3}$, and $P_{4}$ (Fig. 3). $P_{1}$ is an oscillatory mode in medium and high velocity ranges, but changes to a non-oscillatory mode in a low velocity range. In the medium-high velocity range, the damped natural angular frequency of $P_{1}$ does not vary, and the relative degree of stability of $P_{1}$ is high because of the large damping ratio. $P_{2}$ of type II Doubles corresponds to the oscillatory mode $P_{3}$ of type I Doubles, and the damped natural angular frequency and damping ratio of $P_{2}$ of type II Doubles have trends similar to those of $P_{3}$ of type I Doubles with increasing forward velocity. $P_{3}$ and $P_{4}$ of type II Doubles are oscillatory modes in the medium-high velocity range, and changes to non-oscillatory modes in the low velocity range. The damped natural angular frequency of both modes increases with forward velocity in the medium-high velocity range, while their damping ratio decreases.

Types I and II Doubles are vehicles added a semitrailer or full-trailer unit to tractor-semitrailer combinations. Therefore, it is of technical interest to compare the roots of types I and II Doubles and those of tractor-semitrailer or truck-full-trailer combinations. Figures 4 and 5 plot the roots of a domestic tractor-semitrailer combination and a domestic truck-full-trailer combination for maximum loading as a standard condition. The loading conditions in both figures are those usually used in Japan.

$P_{1}$ of the tractor-semitrailer combination is a motion mode with regard to the tractor, and changes from a non-oscillatory mode to an oscillatory mode with increasing forward 
velocity (Fig. 4). $P_{2}$ of this combination is an oscillatory mode dominated by the pendulum motion of semitrailer.

$P_{1}$ of the truck-full-trailer combination is a non-oscillatory mode regarding the truck (Fig. 5). $P_{2}$ and $P_{3}$ are motion modes on the full-trailer and indicate non-oscillatory modes in the low velocity range and oscillatory modes in the medium-high velocity range.

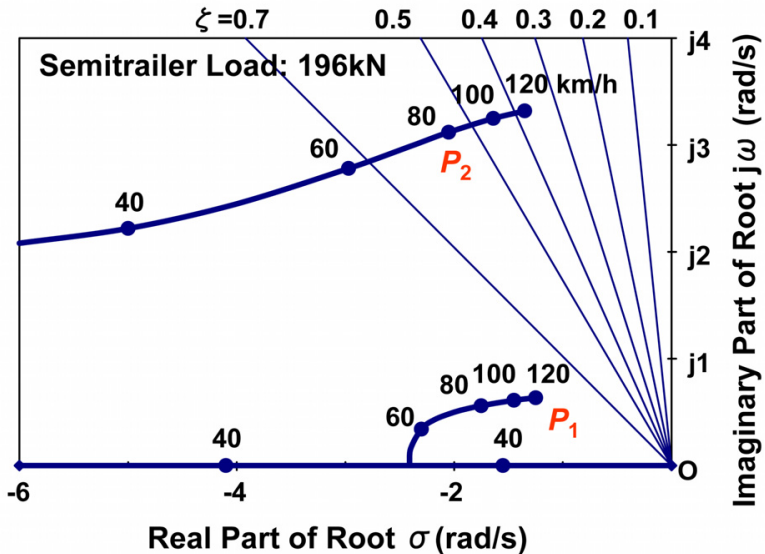

Fig. 4 Roots of tractor and semitrailer combination (standard loading condition)

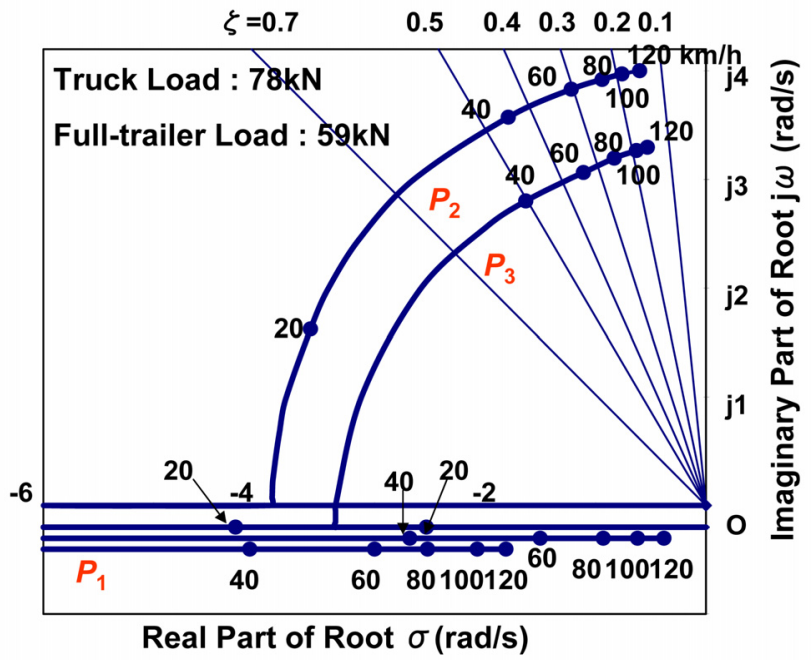

Fig. 5 Roots of truck and full-trailer combination (standard loading condition)

In the next step, the motion modes of type I and II Doubles in Figs. 2 and 3 are compared with those of the tractor-semitrailer and truck-full-trailer combinations in Figs. 4 and 5. $P_{1}$ and $P_{3}$ in Fig. 2 correspond to $P_{1}$ and $P_{2}$ in Fig. 4, and $P_{2}$ in Fig. 2 is a particular oscillatory mode of type I Doubles. $P_{1}$ and $P_{2}$ in Fig. 3 parallel $P_{1}$ and $P_{2}$ in Fig. 4, while $P_{3}$ and $P_{4}$ in Fig. 3 correspond to $P_{2}$ and $P_{3}$ in Fig. 5. Consequently, the motion modes of type I Doubles consist of those of tractor-semitrailer combinations and a particular motion mode of type I Doubles. However, the motion modes of type II Doubles are composed of those of tractor-semitrailer combinations and the portions of truck-full-trailer combinations.

It is also necessary to examine the oscillatory stability of articulated vehicles regarding motion modes of the lowest degree of stability. The damping ratio of type I Doubles is less than that of the tractor-semitrailer combination, and greater than that of the truck-full-trailer combination. The damping ratio of type II Doubles is also less than that of the tractor-semitrailer combination, and equivalent to that of the truck-full-trailer combination. 
In contrast, the damping ratio of type I Doubles exceeds that of type II Doubles. Consequently, type I Doubles seems to excel over type II Doubles as an articulated vehicle type regarding oscillatory stability. Based on the results of the above investigation, the effect of vehicle parameters of type I Doubles on oscillatory stability is examined below.

Figures 6,7 , and 8 plot the calculated results regarding design parameters significantly affecting the oscillatory stability of type I Doubles. Each parameter is fixed, with the exceptions of changing parameters and cornering stiffness, which varied according to the change of axle vertical load.

Figure 6 plots the characteristic roots on variation of the longitudinal position of the center of gravity of the first semitrailer. The damping ratio of $P_{2}$ in the fore is almost equivalent to that in the standard, while that in the aft is considerably reduced in the medium-high velocity range, and the relative degree of stability decreases. Although the calculated results of $c_{1} / l_{1}=0.9$ do not appear in Fig. $6, P_{2}$ becomes unstable at $80 \mathrm{~km} / \mathrm{h}$.

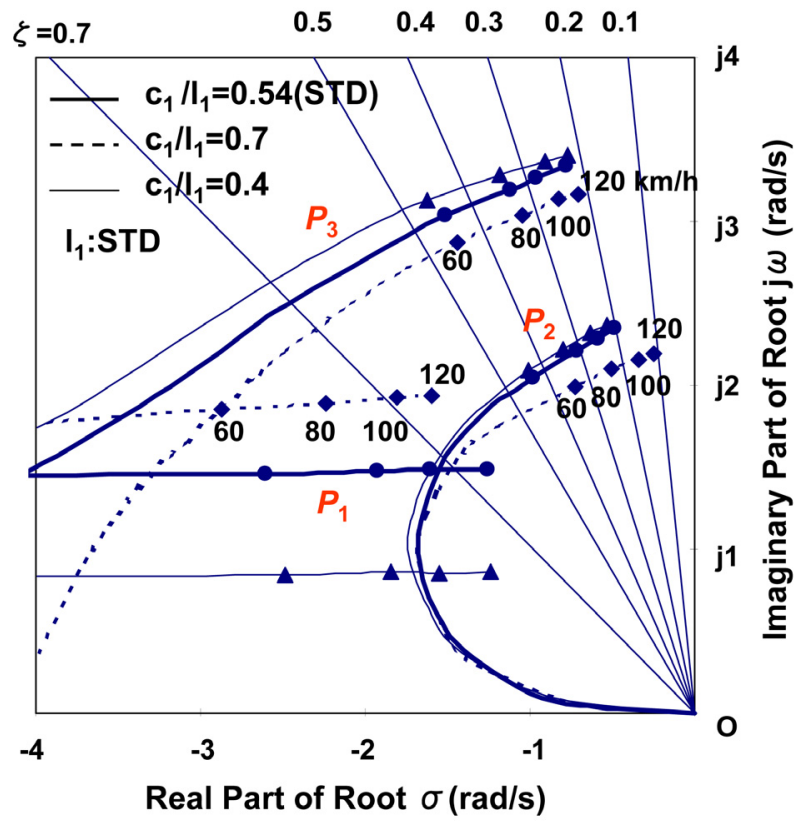

Fig. 6 Effect of moving longitudinal position of the center of gravity of the first semitrailer

Figure 7 depicts the characteristic roots for variation of the longitudinal position of the center of gravity of the second semitrailer. The damping ratio of $P_{2}$ in the fore is slightly reduced in comparison with that in the standard, while that in the aft increases in the medium-high velocity range, and the relative degree of stability increases. That of oscillatory mode $P_{3}$ in the fore is nearly equivalent to that in the standard, while that in the aft is extremely diminished in the medium-high velocity range, and the relative degree of stability decreases. Although the calculated results of $c_{2} / l_{2}=1.0$ do not appear in Fig. 7, $P_{3}$ becomes divergent at velocities exceeding $100 \mathrm{~km} / \mathrm{h}$. 


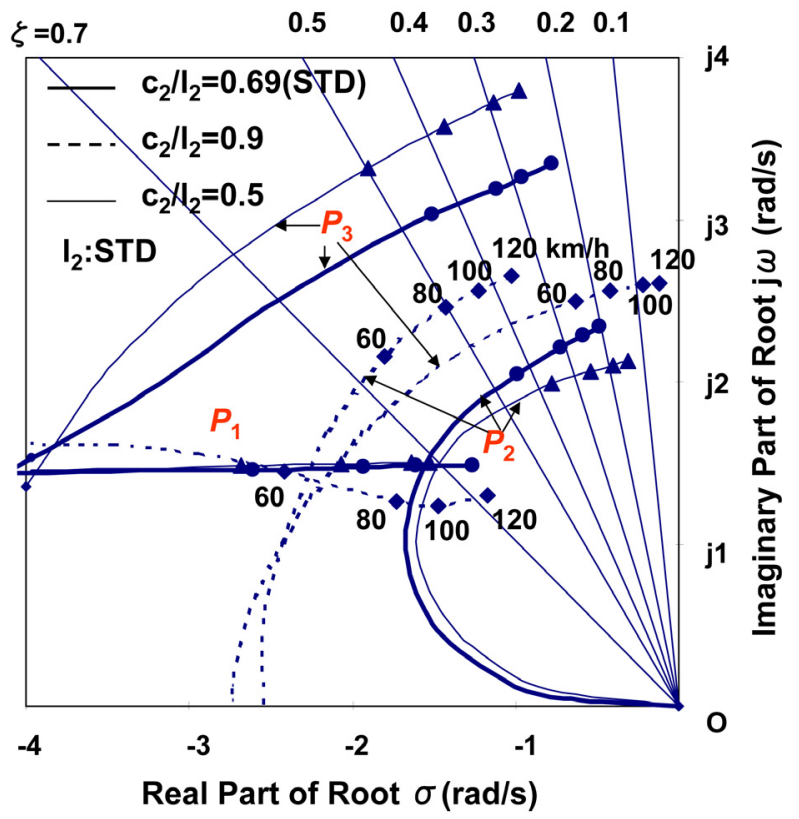

Fig. 7 Effect of moving longitudinal position of the center of gravity of the second semitrailer

Figure 8 plots the characteristic roots for variation of the longitudinal position of the rear fifth-wheel center (rear articulation point) of the first semitrailer. This parameter mainly affects $P_{2}$; the damping ratio in the fore increases slightly swells, while that in the aft is considerably reduced, and the relative degree of stability decreases.

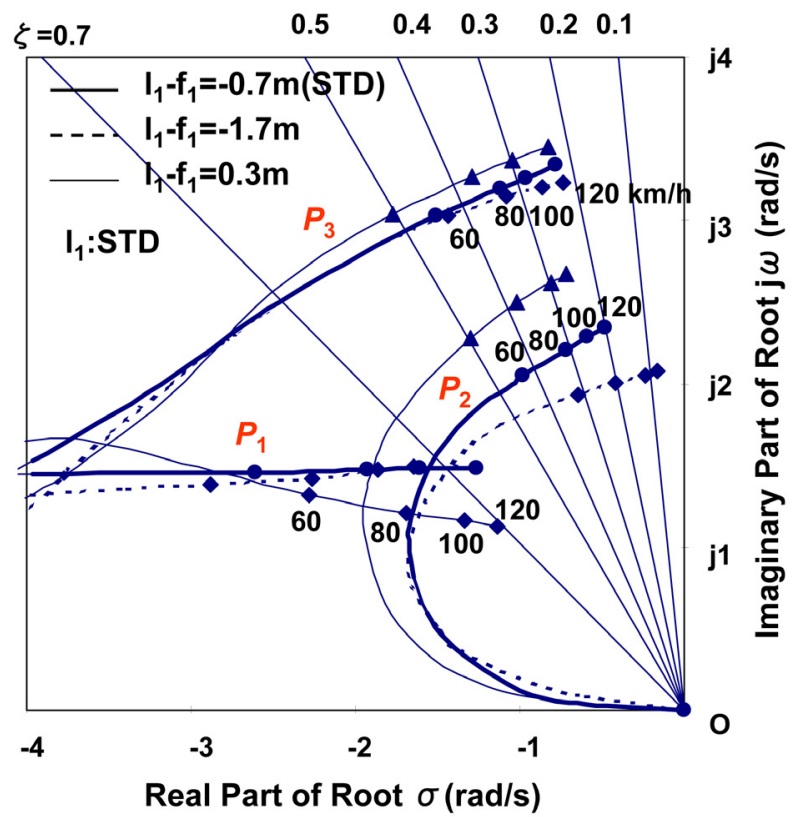

Fig. 8 Effect of moving longitudinal position of the rear fifth-wheel center of the first semitrailer

\subsection{Steering Sensitivity in Steady-state Turning}

Figure 9 indicates the steering sensitivity in steady-state turning with regard to types I and II Doubles, a domestic tractor-semitrailer combination and a domestic truck-full-trailer combination. In this figure, the stability factor of each articulated vehicle is designated. The steering properties of types I and II Doubles, and the tractor-semitrailer combination are weak understeer, and the non-oscillatory mode of those vehicle combinations is kept stable. 
However, the truck-full-trailer combination has weak oversteer, while the critical velocity is very high.

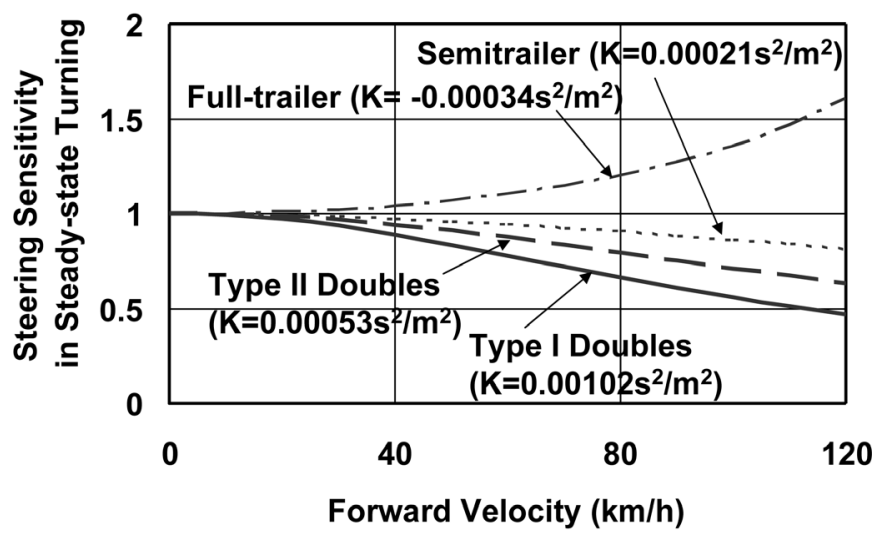

Fig. 9 Steering sensitivity of vehicle combinations in steady-state turning

The design parameters regarding the stability factor which determines steering sensitivity in steady-state turning, of types I and II Doubles are indicated in Eqs. (9) and (11). However, the front articulation point load of the dolly in type II Doubles is usually zero or very small. Therefore, the steering sensitivity of type II Doubles is almost equivalent to that of a tractor-semitrailer combination.

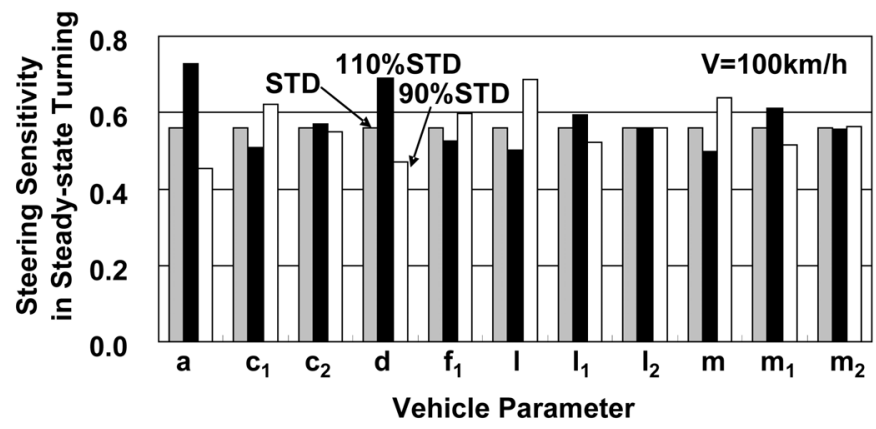

Fig. 10 Parameter study of steering sensitivity of type I Doubles in steady-state turning

Figure 10 presents the calculated result for $100 \mathrm{~km} / \mathrm{h}$ with regard to the effect of the design parameters of type I Doubles on steering sensitivity. Each parameter (except changing and subordinate parameters) is fixed, and the varied percentages from standard conditions are +10 and -10 . It can be seen that the parameters regarding the tractor and first semitrailer mainly affect steering sensitivity.

\subsection{Off-tracking in Steady-state Turning}

Figure 11 indicates the maximum deviation in steady-state turning of $300 \mathrm{~m}$ radius with regard to types I and II Doubles, a domestic tractor-semitrailer combination and a domestic truck-full-trailer combination. The maximum deviation of each vehicle combination decreases from a kinematic deviation at very low velocities as the velocity increases in the low-velocity range and has the minimum values at $40 \mathrm{~km} / \mathrm{h}$, but increases as the velocity increases above that. The maximum deviations of each vehicle become small in the order of type I Doubles, type II Doubles, the truck-full-trailer combination and the tractor-semitrailer combination in the forward velocity range of 80 to $120 \mathrm{~km} / \mathrm{h}$. However, the deviations of types I and II Doubles, and the truck-full-trailer combination is almost the same. 


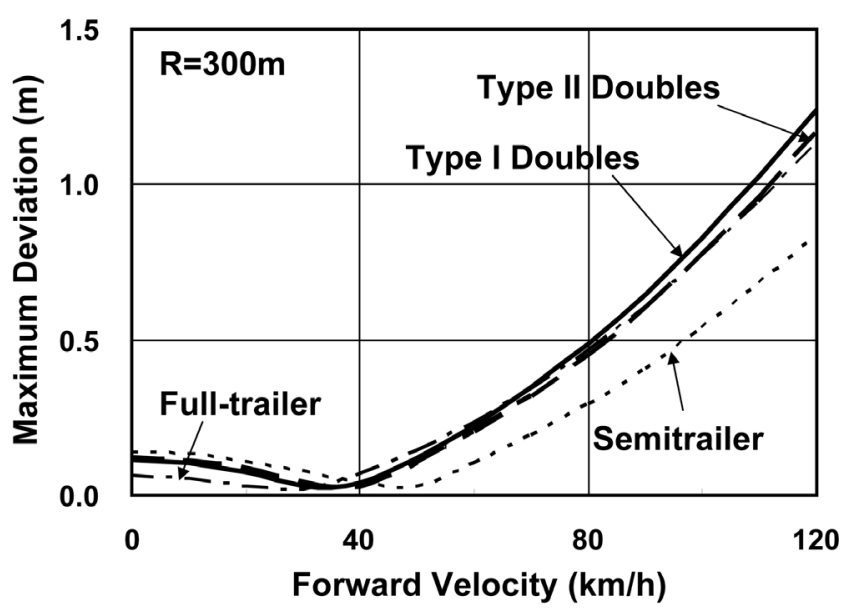

Fig. 11 Off-tracking of vehicle combinations in steady-state turning

Figure 12 presents the calculated result for the effect of design parameters of type I Doubles on the maximum deviation at $100 \mathrm{~km} / \mathrm{h}$. The calculating conditions are equal to those regarding steering sensitivity. In this figure, the effect on the longitudinal position of the rear fifth-wheel center of the first semitrailer is comparatively large. While the calculation of type II Doubles is not addressed in this paper, the result is almost equivalent to that of type I Doubles, and the difference from type I Doubles is that the effect is small on the center of gravity, the distance between front and rear articulation points, and the mass of the dolly; it is somewhat large on the distance between the front articulation point and the center of the axle of the dolly.

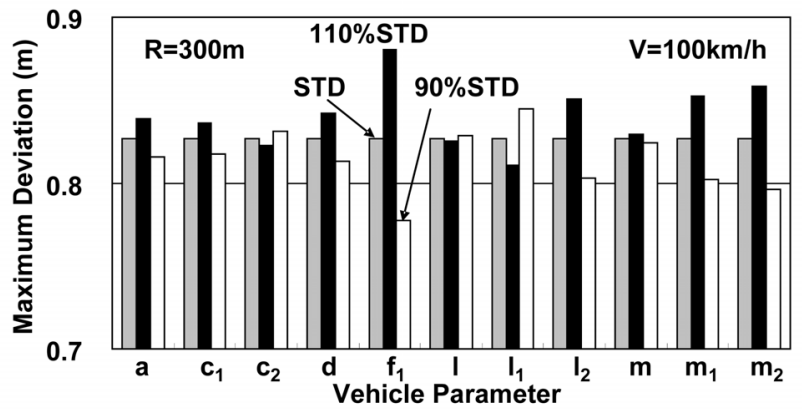

Fig. 12 Parameter study of off-tracking of type I Doubles in steady-state turning

\section{Conclusion}

This paper investigates types of multi-articulated vehicles not used in Japan, on the supposition that these vehicle combinations will be introduced. The subject of this study is tractor and double-trailer combinations consisting of a semitrailer or full-trailer connected to a tractor-semitrailer combination. Basic performance regarding the directional stability and controllability is analyzed and numerically calculated using simplified mathematical models. These two vehicle combinations are designated as types I and II Doubles.

The conclusions are as follow.

(1) The criterion of non-oscillatory stability of types I and II Doubles is a function of the stability factor as well as that of tractor-semitrailer combinations.

(2) Type I Doubles is more stable than type II Doubles in oscillatory modes.

(3) Type I Doubles differs slightly from type II Doubles regarding non-oscillatory stability, steering sensitivity, and off-tracking in steady-state turning. 


\section{Appendix 1 Highest-order Coefficient of Characteristic Equations}

The sign of coefficients $k_{6}$ and $k_{8}$ is independent of forward velocity and always positive based on calculations using the design parameters.

(1) $k_{6}$ of type I Doubles is expressed as

$$
\begin{aligned}
k_{6} & =\left|\begin{array}{cccc}
a_{110} & b_{110} & c_{121} & c_{122} \\
a_{210} & b_{210} & 0 & 0 \\
a_{310} & b_{310} & c_{321} & c_{322} \\
a_{410} & b_{410} & c_{421} & c_{422}
\end{array}\right| \\
& =V\left[\begin{array}{l}
M I I_{1}^{\prime} I_{2}^{\prime}+M T d I_{1}^{\prime} I_{2}^{\prime}-T^{2} I_{1}^{\prime} I_{2}^{\prime}-T_{1}^{2} I I_{2}^{\prime}-T_{2}^{2} I I_{2}^{\prime} \\
-M f_{1}^{2} T_{2}^{2} I+2 T_{1} T_{2}^{2} f_{1} I-T T_{2}^{2} d I_{1}^{\prime}-T T_{1}^{2} d I_{2}^{\prime} \\
-M T T_{2}^{2} d f_{1}^{2}+T^{2} T_{2}^{2} f_{1}^{2}+2 T T_{1} T_{2}^{2} d f_{1}
\end{array}\right],
\end{aligned}
$$

where

$$
M=m+m_{1}+m_{2}, T=m d, T_{1}=m_{1} c_{1}+m_{2} f_{1}, T_{2}=m_{2} c_{2}, I_{1}^{\prime}=I_{1}+m_{1} c_{1}^{2}+m_{2} f_{1}^{2}, I_{2}^{\prime}=I_{2}+m_{2} c_{2}^{2} .
$$

(2) $k_{8}$ of type II Doubles is expressed as

$$
\begin{aligned}
& k_{8}=\left[\begin{array}{llccc}
a_{110} & b_{110} & c_{121} & c_{122} & c_{123} \\
a_{210} & b_{210} & 0 & 0 & 0 \\
a_{310} & b_{310} & c_{321} & c_{322} & c_{323} \\
a_{410} & b_{410} & c_{421} & c_{422} & c_{423} \\
a_{510} & b_{510} & c_{521} & c_{522} & c_{523}
\end{array} \mid\right. \\
&=V\left[\begin{array}{l}
M I I_{1}^{\prime} I_{2}^{\prime} I_{3}^{\prime}+M T d I_{1}^{\prime} I_{2}^{\prime} I_{3}^{\prime}-M T_{3}^{2} f_{2}^{2} I I_{1}^{\prime}-M T_{3}^{2} f_{1}^{2} I I_{2}^{\prime}-M T_{2}^{2} f_{1}^{2} I I_{3}^{\prime} \\
+2 M T_{2} T_{3}^{2} f_{1}^{2} f_{2} I-M T T_{3}^{2} d f_{2}^{2} I_{1}^{\prime}-M T T_{3}^{2} d f_{1}^{2} I_{2}^{\prime}-M T T_{2}^{2} d f_{1}^{2} I_{3}^{\prime} \\
+2 M T T_{2} T_{3}^{2} d f_{1}^{2} f_{2}-T_{1} T_{1} I I_{2}^{\prime} I_{3}^{\prime}-T_{3}^{2} I I_{1}^{\prime} I_{2}^{\prime}-T_{2}^{2} I I_{1}^{\prime} I_{3}^{\prime}-T^{2} I_{1}^{\prime} I_{2}^{\prime} I_{3}^{\prime} \\
+2 T_{2} T_{3}^{2} f_{2} I I_{1}^{\prime}+2 T_{1} T_{3}^{2} f_{1} I I_{2}^{\prime}+2 T_{1} T_{2}^{2} f_{1} I I_{3}^{\prime}-T T_{1}^{2} d I_{2}^{\prime} I_{3}^{\prime}-T T_{2}^{2} d I_{1}^{\prime} I_{3}^{\prime} \\
-T T_{3}^{2} d I_{1}^{\prime} I_{2}^{\prime}+T^{2} T_{3}^{2} f_{2}^{2} I-4 T_{1} T_{2} T_{3}^{2} f_{1} f_{2} I+2 T T_{2} T_{3}^{2} d f_{2} I_{1}^{\prime} \\
+2 T T_{1} T_{3}^{2} d f_{1} I_{2}^{\prime}+2 T T_{1} T_{2}^{2} d f_{1} I_{3}^{\prime}+T^{2} T_{3}^{2} f_{2}^{2} I_{1}^{\prime}+T^{2} T_{3}^{2} f_{1}^{2} I_{2}^{\prime} \\
+T^{2} T_{2}^{2} f_{1}^{2} I_{3}^{\prime}+T T_{1}^{2} T_{3}^{2} d f_{2}^{2}-4 T T_{1} T_{2} T_{3}^{2} d f_{1} f_{2}-2 T^{2} T_{2} T_{3}^{2} f_{1}^{2} f_{2}
\end{array}\right]
\end{aligned}
$$

where

$$
\begin{aligned}
& M=m+m_{1}+m_{2}+m_{3}, T=m d, T_{1}=m_{1} c_{1}+\left(m_{2}+m_{3}\right) f_{1}, T_{2}=m_{2} c_{2}+m_{3} f_{2}, T_{3}=m_{3} c_{3}, \\
& I^{\prime}=I_{1}+m_{1} c_{1}^{2}+\left(m_{2}+m_{3}\right) f_{1}^{2}, I_{2}^{\prime}=I_{2}+m_{2} c_{2}^{2}+m_{3} f_{2}^{2}, I_{3}^{\prime}=I_{3}+m_{3} c_{3}^{2} .
\end{aligned}
$$

Appendix 2 Vehicle Specifications used in Numerical Calculations (Standard Loading Conditions)

(1) Type I Doubles

$$
\begin{aligned}
& a=1.167 \mathrm{~m}, b=2.333 \mathrm{~m}, c_{1}=2.853 \mathrm{~m}, c_{2}=3.669 \mathrm{~m}, d=1.983 \mathrm{~m}, f_{1}=6.000 \mathrm{~m}, \\
& l=3.500 \mathrm{~m}, l_{1}=5.300 \mathrm{~m}, l_{2}=5.300 \mathrm{~m}, \mathrm{~m}=6000 \mathrm{~kg}, m_{1}=13000 \mathrm{~kg}, m_{2}=13000 \mathrm{~kg}, \\
& I=19600 \mathrm{kgm}^{2}, I_{1}=68600 \mathrm{kgm}^{2}, I_{2}=58800 \mathrm{kgm}^{2}, C_{f}=186 \mathrm{kN} / \mathrm{rad}, \\
& C_{r}=332 \mathrm{kN} / \mathrm{rad}, C_{1}=405 \mathrm{kN} / \mathrm{rad}, C_{2}=370 \mathrm{kN} / \mathrm{rad} .
\end{aligned}
$$

(2) Type II Doubles 
$a=1.122 m, b=2.078 m, c_{1}=2.866 m, c_{2}=1.800 m, c_{3}=2.866 m, d=1.778 m$,

$f_{1}=6.200 \mathrm{~m}, f_{2}=2.000 \mathrm{~m}, l=3.200 \mathrm{~m}, l_{1}=5.400 \mathrm{~m}, l_{2}=2.000 \mathrm{~m}, l_{3}=5.400 \mathrm{~m}$,

$m=5700 \mathrm{~kg}, m_{1}=13000 \mathrm{~kg}, m_{2}=1000 \mathrm{~kg}, m_{3}=13000 \mathrm{~kg}, I=14700 \mathrm{kgm}^{2}$,

$I_{1}=67200 \mathrm{kgm}^{2}, I_{2}=686 \mathrm{kgm}^{2}, I_{3}=67200 \mathrm{kgm}^{2}, C_{f}=181 \mathrm{kN} / \mathrm{rad}$,

$C_{r}=344 \mathrm{kN} / \mathrm{rad}, C_{1}=333 \mathrm{kN} / \mathrm{rad}, C_{2}=333 \mathrm{kN} / \mathrm{rad}, C_{3}=331 \mathrm{kN} / \mathrm{rad}$.

(3) Tractor-semitrailer Combination

$a=2.133 m, b=1.867 m, c=4.556 m, d=1.667 m, l=4,000 m, l_{1}=8.200 m$,

$m=7500 \mathrm{~kg}, m_{1}=27000 \mathrm{~kg}, I=29400 \mathrm{kgm}^{2}, I_{1}=254800 \mathrm{kgm}^{2}$,

$C_{f}=178 \mathrm{kN} / \mathrm{rad}, C_{r}=695 \mathrm{kN} / \mathrm{rad}, C_{1}=687 \mathrm{kN} / \mathrm{rad}$.

(4) Truck-full-trailer Combination

$a=3.310 \mathrm{~m}, b=1.490 \mathrm{~m}, c_{1}=2.500 \mathrm{~m}, c_{2}=2.059 \mathrm{~m}, d=3.290 \mathrm{~m}, f_{1}=2.500 \mathrm{~m}$,

$l=4.800 \mathrm{~m}, l_{1}=2.500 \mathrm{~m}, l_{2}=3.500 \mathrm{~m}, m=14500 \mathrm{~kg}, m_{1}=1000 \mathrm{~kg}, m_{2}=8500 \mathrm{~kg}$,

$I=78400 \mathrm{kgm}^{2}, I_{1}=588 \mathrm{kgm}^{2}, I_{2}=24500 \mathrm{kgm}^{2}, C_{f}=185 \mathrm{kN} / \mathrm{rad}$,

$C_{r}=385 \mathrm{kN} / \mathrm{rad}, C_{1}=185 \mathrm{kN} / \mathrm{rad}, C_{2}=193 \mathrm{kN} / \mathrm{rad}$.

\section{References}

(1) Dugoff, H. and Murphy, R. W., The Dynamic Performance of Articulated Highway Vehicles - A Review of the State-of-the-Art, SAE Transactions, Vol. 80 (1971), pp.897-906.

(2) Vlk, F., Lateral Dynamics of Commercial Vehicle Combinations - A Literature Survey, Vehicle System Dynamics, 11 (1980), pp. 305-324.

(3) Aoki, A., Marumo, Y., Kageyama, I. and Kuwahara, K., Controllability and Loading Conditions of Tractor and Semitrailer Combinations in Lane Change (in Japanese), Transactions of the Japan Society of Mechanical Engineers, Series C, Vol.75, No.749 (2009), pp. 66-73. 